\title{
La reserva cognitiva como prevención en el deterioro de las funciones neurocognitivas en la vejez
}

\author{
Cognitive reserve as prevention in the deterioration of neurocognitive functions in old age
}

Reserva cognitiva como prevenção na deterioração das funções neurocognitivas na velhice

Pablo Andrés Barba Gallardo

pablo.barba@utc.edu.ec

https://orcid.org/0000-0003-2563-9081

Universidad Técnica de Cotopaxi, Latacunga - Ecuador

\section{RESUMEN}

La investigación se desarrolló bajo los antecedentes de los altos índices de personas con problemas neuropsicológicos y neurodegenerativos en la edad adulta. Para la consecución del objetivo el cual fue identificar los mecanismos preventivos de la reserva cognitiva en los procesos neurodegenerativos normales y patológicos, desde el enfoque educacional; la investigación fue no experimental de tipo descriptiva; se trabajó con 37 personas (19 mujeres y 18 hombres) comprendidos entre 60 y 78 años. Las técnicas utilizadas son la observación, entrevistas y aplicación de instrumentos clínicos psicométricos: MMSE, CRC, MoCA, además, de entrevistas a expertos. Los factores predominantes de la reserva cognitiva que inciden en los mecanismos preventivos son la estimulación neurocognitiva, experiencias vividas, educación, ocupación y tipo de ocio, teniendo impactos directos en la neuropsicología de las personas. Concluyendo que entrenar y dotar al cerebro sobre procesos de habilidad cognitiva son de impacto positivo para las personas en su envejecimiento.

Palabras clave: Reserva cognitiva; Patológico; Neuropsicológico; Neurodegenerativo; Neurocognitivo

\section{ABSTRACT}

The research was developed against the background of high rates of people with neuropsychological and neurodegenerative problems in adulthood. To achieve the objective which was to identify the preventive mechanisms of the cognitive reserve in normal and pathological neurodegenerative processes, from the educational approach; the research was non-experimental of a descriptive type; We worked with 37 people (19 women and 18 men) between 60 and 78 years old. The techniques used are observation, interviews and application of clinical psychometric instruments: MMSE, CRC, MoCA, in addition to interviews with experts. The predominant factors of the cognitive reserve that affect the preventive mechanisms are neurocognitive stimulation, lived experiences, education, occupation and type of leisure, having direct impacts on the neuropsychology of people. Concluding that training and equipping the brain with cognitive ability processes have a positive impact for people in their aging.

Key words: Cognitive reserve; Pathological; Neuropsychological; Neurodegenerative; Neurocognitive

\section{RESUMO}

A pesquisa foi desenvolvida tendo como pano de fundo altos índices de pessoas com problemas neuropsicológicos e neurodegenerativos na idade adulta. Alcançar o objetivo que foi identificar os mecanismos preventivos da reserva cognitiva em processos neurodegenerativos normais $\mathrm{e}$ patológicos, a partir da abordagem educativa; a pesquisa foi não experimental do tipo descritiva; trabalhamos com 37 pessoas (19 mulheres e 18 homens) entre 60 e 78 anos. As técnicas utilizadas são observação, entrevistas e aplicação de instrumentos clínicos psicométricos: MMSE, CRC, MoCA, além de entrevistas com especialistas. Os fatores predominantes da reserva cognitiva que afetam os mecanismos preventivos são estimulação neurocognitiva, experiências vividas, educação, ocupação e tipo de lazer, tendo impactos diretos na neuropsicologia das pessoas. Concluir que treinar e equipar o cérebro com processos de habilidades cognitivas tem um impacto positivo para as pessoas no envelhecimento.

Palavras-chave: Reserva cognitiva; Patológica; Neuropsicológica; Neurodegenerativa; Neurocognitiva 


\section{INTRODUCCIÓN}

En la actualidad, somos testigos de un creciente envejecimiento de la población, lo que supone un incremento notable de enfermedades ligadas a la edad, tanto, físicas como neuropsicológicas, como es el caso de las demencias (Rodríguez y Sánchez, 2004). En concreto, las demencias y el Deterioro Cognitivo Leve (DCL), son los procesos más incapacitantes para las personas que lo padecen, es especial los adultos y adultos mayores, ya que conlleva una pérdida progresiva de habilidades, llegando a producir una reducción de las actividades placenteras y/o cotidianas, traduciéndose en una disminución de la calidad de vida.

La calidad de vida se define como la percepción personal que un individuo tiene de su situación vital. Dentro de los factores que pueden influir en la CV, se encuentra la Reserva Cognitiva (RC), que podría entenderse como la capacidad del cerebro para hacer frente al daño cerebral generado por la patología o el envejecimiento propio del desarrollo, mediante procesos cognitivos preexistentes $\mathrm{o}$ compensatorios que se adquirió a través de su vida.

Desde una perspectiva comportamental, la RC podría entenderse como la capacidad del cerebro para hacer frente al daño cerebral generado por la patología, mediante procesos cognitivos preexistentes o compensatorios (Stern, 2002), desarrollados a partir de cierto nivel de actividad mental que la persona ha ido llevando a cabo a lo largo de su vida, lo que le permite retrasar la manifestación clínica de los síntomas demenciales y los propios de la edad. (Valenzuela, 2008).

Relacionando este constructo con la autopercepción que los pacientes manifiestan acerca de su calidad de vida, Inouye y Pedrazzani
(2007), llevaron a cabo un estudio exploratorio, encontrando que los niveles más altos de escolaridad y la participación frecuente en actividades físicas, se asociaban a una autovaloración más elevada del bienestar personal de la población mayor. No obstante, otras investigaciones con objetivos similares, pero en las que se empleó únicamente muestras de sujetos con bajo nivel educacional, hallaron que la valoración de la R.C., resultaba poco estructurada generando riegos en casos de envejecimiento y patología. (Zea Herrera et al., 2008).

Según la investigación desarrollada, desde los modelos activos de RC, existen múltiples variables que influyen en su desarrollo, mantenimiento y potenciación, a lo largo del ciclo vital. Aunque no existen estudios concluyentes en relación con la relevancia de cada uno de los componentes, así como la combinación más adecuada de la medida, para su operacionalización se han utilizado variables como educación, ocupación, y actividades de ocio: físicas, mentales y sociales, que han sido las más empleadas, obteniendo resultados concluyentes.

En este sentido, uno de los retos de la presente investigación fue analizar los mecanismos de RC en los grupos de adultos y adultos mayores, y asociar a la prevención de los procesos neurocognitivos del envejecimiento normal y patológico, así como analizar las similitudes y diferencias en adultos con deterioro cognitivo, puesto que el estudio se asocia a la formación de la RC como preventor de los procesos de diversas enfermedades demenciales, dado que la RC podría modificar el curso de la enfermedad (Stern, 2002).

De este modo, en este trabajo se desarrolló una medida de RC, siguiendo el procedimiento descrito 
por autores como Scarmeas (2007); Solé-Padullés et al. (2009), Stern (2002). A partir de la obtención de la medida de RC se compararon sujetos con alta reserva cognitiva (ARC) y baja reserva cognitiva (BRC), así como sujetos con BRC y deterioro cognitivo.

La reserva cognitiva es un tema que está en desarrollo en las investigaciones científicas a nivel nacional e internacional, existe todavía cierta incredulidad sobre los mecanismos cerebrales y la estimulación dela Reserva Cognitiva para confrontar los procesos degenerativos normales y patológicos del envejecimiento, las pocas investigaciones realizadas apuntan a causales de funcionalidad, tipo, variabilidad y frecuencia de patologías, o a su vez, a la rehabilitación y alternativas clínicas para reducir los síntomas propios de las enfermedades, por lo que analizar los aspectos preventivos y estimulantes de la R.C. tiene efectos positivos en el quehacer clínico.

La importancia de generar resultados clínicos significativos a nivel neurocognitivo para el beneficio de los pacientes de los grupos de adultos y adultos mayores, conlleva un trabajo técnico que permite entender el dinamismo cerebral de este grupo etario y, a su vez, se puede acceder a otros espacios del mismo cerebro en donde se encuentra solución procedimental al problema. El tema es interdisciplinario, los criterios clínicos de la comunidad de la salud es primordial, de este modo, desde la psicología y neuropsicología se ha observado que las personas con patologías cognitivas, especialmente demencias y envejecimiento con declives, tienen condiciones que deterioran su integridad, que no existe el manejo adecuado e incluso son excluidos y aislados, sin tener la oportunidad de sobrellevar los cuadros sintomáticos presentados; es claro comprender que el trabajo de prevención es más efectivo en los inicios de la enfermedad, cuando existe las primeras manifestaciones patógenas o de los síntomas propios de la edad.

Con estos resultados y generando un adecuado diagnóstico apegado al origen del problema se podrán diseñar los mecanismos de tratamiento efectivos en el campo neuropsicológico. Con la investigación se pretende reducir el impacto patógeno y degenerativo que atemoriza a las personas en edades avanzadas y a sus familiares, donde se muestran alteraciones emocionales en los procesos de la enfermedad en relación a sus seres queridos y/o familiares.

\section{MÉTODO}

La investigación es descriptiva de corte transversal, se sustenta en estudios bibliográficos, diagnósticos, documentales y de campo, para el planteamiento epidemiológico del deterioro de las neurofunciones cerebrales. Se trabajó en el centro de adultos mayores y el grupo de jubilados respectivamente, con un grupo de 37 personas (19 mujeres y 18 hombres) comprendidas entre 64 y 78 años, (zona sur-centro del Distrito Metropolitano de Quito), provenientes de diversos contextos y realidades socioculturales, además se obtuvo preliminares de familiares aleatoriamente, dicha información se complementó con la entrevista de los investigados. Son personas que no padecen ninguna patología o antecedentes neuropsicológicos relevantes (ejemplo: demencias, accidentes cerebrovasculares (ACV), traumatismos craneoencefálicos severos o de consideración, consumo de psicotrópicos), confirmando que cumplieran los criterios de inclusión. 
Se entregó el perfil de estudio y se puntualizó en la ejecución de firmas para el acuerdo de consentimiento informado, que posteriormente fue aprobado por el Comité Dirigencial de Jubilados.

Para la obtención de información del estudio se lo realizó in situ, lo cual, integra como objeto de investigación a personas adultas y adultas mayores, además, de familiares, investigadores universitarios, profesionales de la salud de instituciones geriátricas y casas de salud, para generar la triangulación de la información de los diferentes contextos de las personas estudiadas. Se realizó una serie de actividades dinámicas y de neuroestimulación la cual sirvió para dos ejes, identificación de funciones neuropsicológicas de curso normal y patológico, $\mathrm{y}$ de estimulación de los reservorios cognitivos aprendidos en su evolución; la cual nos permitió elaborar el plan de prevención e intervención para los procesos mencionados.

En primera instancia, se utilizó el Mini-Mental State Examination (MMSE), en la versión original de Folstein (1975) para posteriormente generar comparación internacional con resultados en otras investigaciones. El objetivo de éste instrumento comprende la realización de algunas acciones por parte de la persona evaluada. Sus resultados permiten tener una valoración de cribado o despistaje de su estado cognitivo en distintas áreas que se pueden relacionar con diferentes síntomas cognitivos, que en el MMSE son los siguientes: a) Orientación temporal y espacial; b) Memoria inmediata $y$ retención; c) Concentración y memoria de trabajo; d) Lenguaje, y; e) Praxis constructiva gráfica. El MMSE de Folstein (1975) cuenta con un rango de puntuación de 0 a 30, según la puntuación obtenida los grados de deterioro son: 27 y 30 ausencia de deterioro cognitivo, 26 y 25 consideraciones sobre un posible deterioro cognitivo, 24 y 10 existencia de deterioro cognitivo de leve a moderado.

En segunda instancia, se aplicó el Cuestionario de Reserva Cognitiva (CRC) de 8 ítems, mediante el uso de cuartiles, por lo cual, una puntuación igual o menor de 6 puntos, que pertenece al cuartil $1(\leq \mathrm{C} 1)$, situaría el grado de reserva cognitiva del sujeto en el rango inferior. Entre 7 y 9 puntos (C1C2) correspondería a una reserva cognitiva situada en el rango medio-bajo, mientras que entre $10 \mathrm{y}$ 14 (C2-C3) se consideraría medio-alto. Aquellas puntuaciones $\geq 15$ puntos se clasificarían como una reserva cognitiva situada en la categoría superior $(\geq \mathrm{C} 4)$.

En tercera instancia, se administró la Evaluación Cognitiva Montreal (Montreal Cognitive Assessment-MoCA), desarrollado por Nasreddine et al., en el año 2005, versión en español, que consiste en un instrumento de screening para detectar disfunciones cognitivas, a través de la evaluación de habilidades como la atención, concentración, funciones ejecutivas, memoria, lenguaje, capacidades visuoconstructivas, cálculo y orientación.

\section{Análisis estadístico}

La información se analizó con Microsoft Excel y el software estadístico IBM SPSS 22. Los resultados de las características sociodemográficas: edad, género, escolaridad, tipo de trabajo e ingresos de los participantes (Tabla 1) 
Tabla 1. Datos sociodemográficos: edad, género, escolaridad, tipo de trabajo e ingresos.

\begin{tabular}{llll}
\hline & & Datos sociodemográficos & \\
\hline $\mathbf{n}$ & 37 & & \\
Edad & $\leq 64-78 \geq$ & & Masculino 18 \\
Género & Femenino 19 & & \\
Escolaridad & Primaria & $18 \%$ & \\
& Secundaria & $56 \%$ & \\
& Superior & $22 \%$ & \\
& Especialidad & $4 \%$ & \\
Trabajo & Artesanal & $23 \%$ & $\leq 400$ \\
& Oficio & $58 \%$ & $\leq 401-800 \geq$ \\
Ingresos & Profesional & $19 \%$ & $801 \geq$ \\
& Básico & $51 \%$ & SSD \\
& Medio & $38 \%$ & \\
\hline
\end{tabular}

RESULTADOS Y DISCUSIÓN

En el análisis interpretativo del MMSE, se evidencia DCL (Tabla 2), de acuerdo a los rangos de edad $36 \%$, estudio $42.3 \%$ y trabajo $63.7 \%$. Existe evidencia que las personas que tuvieron un estudio superior y trabajo profesional tienen índices bajos de DCL y obviamente rangos estándares altos de normalidad en los procesos cognitivos.

Tabla 2. MMSE.

\begin{tabular}{lcccc}
\hline & Rango & PD & Media & DCL/Nor \\
\hline Edad & $64-69$ & $23-26$ & 27,3 & $6,5 / 10,3$ \\
& $70-74$ & $17-22$ & 63,8 & $17,7 / 41,8$ \\
\multirow{3}{*}{ Estudio } & $75-78$ & $16-18$ & 8,9 & $11,8 / 11,9$ \\
& Pri. & $16-19$ & 18,6 & $17,8 / 22,5$ \\
& Sec. & $19-23$ & 40,2 & $14,3 / 16,2$ \\
& Uni. & $22-25$ & 27,3 & $6,1 / 9,1$ \\
Trabajo & Espec. & $23-26$ & 13,9 & $4,1 / 9,9$ \\
& Art. & $17-22$ & 39,5 & $31,2 / 9,3$ \\
& Ofi. & $16-24$ & 36,7 & $27,7 / 11,2$ \\
& Prof. & $23-26$ & 23,8 & $4,8 / 15,8$ \\
\hline
\end{tabular}


En el análisis del CRC, se detecta que las personas que tuvieron actividades de estudio superior, actividad lectora continua, ocupación laboral profesional o directiva, poseen RC media alta y superior; las actividades de formación musical, cursos preparatorios y juegos intelectuales coadyuvan a una RC media baja y media alta; Formación en estudio primarios, escolaridad de padres es baja y/o nulo en la conservación de RC. Se excluye el ítem idiomas ya que todos hablan únicamente lenguaje materno. (Tabla. 3).

Tabla 3. CRC.

\begin{tabular}{lccc}
\hline \multicolumn{1}{c}{ Cuar. } & $\mathbf{n}^{\circ}$ & $\%$ & R. Cog. \\
\hline$\leq 6 \mathrm{C} 1$ & 17 & 45,9 & Baja \\
$\leq 7-9 \geq \mathrm{C} 1-\mathrm{C} 2$ & 8 & 21,7 & Media baja \\
$\leq 10-14 \geq \mathrm{C} 2-\mathrm{C} 3$ & 9 & 24,3 & Media alta \\
$15 \geq \mathrm{C} 4$ & 3 & 8,1 & Superior \\
\hline
\end{tabular}

En el análisis del test MoCA (Tabla. 4), se concatena a los resultados anteriores y se puede catalogar que los evaluados que tuvieron actividades de estudio superiores y continuos, además de actividades vinculadas al desarrollo cognitivo presentan una normalidad y DCL con puntuaciones mínimas; las personas que no han realizado acciones de estimulación cognitiva en cuanto al tiempo de ejecución, comprensión, verbalización, secuencia y memoria, muestran dificultades e incluso bloqueos en la toma de decisiones.

Tabla 4. MoCA

\begin{tabular}{|c|c|c|}
\hline Media & $\mathrm{n}^{\circ} \%$ & Int. \\
\hline$\leq 26-30$ & $6 / 16.2 \%$ & Nor \\
\hline$\leq 25$ & $31 / 83.8 \%$ & DCL \\
\hline
\end{tabular}

En la aplicación del test se presentaron casos que no entendían ciertas órdenes propias de la ejecución del test, en las que se tuvo que alargar el tiempo de realización, considerando como procesos cognitivos confusos que son propios del deterioro cognitivo.

\section{Análisis de las entrevistas sobre las actividades Psicomotrices y Neuroestimulatorias}

Grupos etarios por género. Se estableció que las mujeres poseen una RC más ampliada, correlacionadas a las actividades de motricidad 
fina y lectura, escritura y hablar con alternativas complejas, prevalentemente existe una Alta Reserva Cognitiva (ARC) 59.2\%. Con relación a los hombres existe una $\mathrm{RC}$ menos expuesta a las actividades de neuroestimulación referentes a motricidad fina y actividades de educación básica comoleer, escribiry hablar con alternativas complejas, prevalentemente existe Baja Reserva Cognitiva (BRC) 40.8\%.

\section{Actividades laborales desarrolladas con análisis}

transversal. Se divide en actividades manuales, oficios y técnicas - profesionales, por lo que, es referente de confiabilidad que las personas en grupo mixto (hombres y mujeres) que se dedicaban a actividades cognitivas o con relación cognitiva, tienen un proceso de RC con un $61.5 \%$, se detalla entre ellas (profesores, secretarias, abogados y administradores); y las personas que tenían actividades de arte y oficio tienen una RC de 33.1\%, entre ellas se detalla a (mecánicos, conductores, recepcionistas, trabajos informales).

Actividades de Ocio. las personas con actividades de ocio productivo como: deportes (Ecuavoley, fútbol, natación, bicicleta, atletismo), viajes, aleatoriamente tienen un RC más flexible con $29 \%$, lo contrario de las personas que han realizado actividades como ver películas, salir al parque, visitas a familiares y reuniones sociales se ubican con menos flexibilidad en un $11 \%$ de la media general.

A continuación, se evidencia las actividades neuroestimulantes realizadas con fines preventivos, se debe aclarar que este procedimiento está condicionado a una estructura y mecanismo de RC que ya viene con la persona, con lo cual se trabaja con los niveles que se posee, esto no es limitante ya que se debe encontrar la actividad propicia para estimular los almacenes cognitivos generadores de actividades anatocerebrales óptimas para el desarrollo.

\section{Actividad de secuencia y coordinación motriz} (Aeróbicos). Se llevó una ficha de observación con el estado de actividad y en la séptima serie registrada después de tres semanas de trabajo se registró un incremento de velocidad de respuestas motrices y cognitivas $22 \%$ más rápida con referencia de la primera serie.

Lectura. Se realizó una serie de lecturas ocupacionales in situ en tiempos establecidos como cortos, además se envió ciertas lecturas para su tiempo de ocio en una serie de 7 veces, y se correlaciono con la función de memoria y los análisis fueros cualitativamente superiores al inicio del ejercicio. Los participantes en un 31\% estuvieron acertados en las preguntas post lecturas; de las 37 personas promovidas al ejercicio, 5 no lograron leer y 6 no tenían capacidad de análisis de la lectura.

Bailar. Es una actividad que gusta mucho al grupo del adulto mayor, además de ser una actividad de alta demanda cognitiva que fomenta procesos complejos en el cerebro; esta actividad fue realiza con estilos de música que generan cierta complejidad, en las cuales se dio las directrices para el seguimiento de la actividad, se obtuvo el $61 \%$ de resultados positivos derivados en coordinación específica en las series propuestas progresivamente.

\section{Discusión}

Muchas intervenciones están direccionadas únicamente al campo clínico, cuando la enfermedad tiene un curso avanzado, limitándose solo a ese procedimiento, y no se especula el antes del 
aparecimiento de la enfermedad, puesto que no se conoce otras alternativas de intervención o procesos preventivos como la reserva cognitiva, que produce cambios significativos en las personas adultas.

La reserva cognitiva es un factor protector y un mecanismo neuropsicológico preventivo en el envejecimiento con declives cognitivos y patológicos. Hay estudios que ratifican que tener etapas óptimas de vida en el desarrollo, en donde haya procesos cognitivos de alta demanda, generan beneficios superlativos en las funciones neurocognitivas. Incluso se ha podido resaltar que las personas que presentan cuadros demenciales por condiciones genéticas o condiciones causales de estilos de vida patógenos, que han logrado desarrollar RC adecuados, el progreso de sus cuadros degenerativos han sido más sostenidos y tratables.

En relación a como la reserva cognitiva actúa sobre valoración de la calidad de vida, los datos del estudio proyectan que los individuos con mayor RC obtuvieron puntuaciones más elevadas, lo cual parece convertirse en una tendencia, poseer mejor dinamismo de la RC estructurada en la evolución de los adultos mayores añadido a la estimulación neurocognitiva, es positivo para sus condiciones de vida. Aunque no se ha encontrado investigaciones que indiquen la relación entre este constructo a nivel global de RC y la CV, pues, si existen publicaciones orientadas al analizar de forma aislada como algunas variables pertenecientes a la $\mathrm{RC}$, se asocian a la calidad de vida percibida, promoviendo conclusiones compatibles con los resultados arrajados.

En este sentido, destaca el estudio de Ferrando, et al., (1996) en el que examinando la percepción del estado de salud en población mayor no institucionalizada, se encontró que las mujeres que peor valoraban su salud eran aquellas con un bajo nivel educativo y falta de apoyo en las actividades cotidianas, sin embargo, en el caso de los varones, la percepción negativa de la salud estaba más asociada a la ausencia de apoyo emocional. Resultados equivalentes en otros estudios donde se ponen de manifiesto asociaciones significativas que relacionan la autovaloración negativa de la calidad de vida con el bajo nivel educacional (Inouye y Pedrazzani, 2007).

En cuanto al estado cognitivo del grupo examinado, se explica que las realidades de cada uno de ellos son distintas, puesto que los temas de estimulación, trabajo y educación en todos sus niveles, hace algunas décadas atrás, no eran estipuladas como importantes, tampoco tenían la suficiente teorización para ser cumplidas; otro factor de análisis es que sus padres o tutores tampoco tuvieron acceso a la educación, proyectando una escaza importancia a que sus hijos tengan una educación básica y en mejores realidades secundaria, dejando como privilegio de muy pocos el acceso a la educación superior; propiciando el trabajo de oficios como fuente primaria de vida desde muy cortas edades, por lo menos ese es el contexto de Ecuador; desde esa óptica las actividades que se desarrollaban no iban más allá de un cumplimiento de subsistencia, sea laboral o casero, entonces, sus aspectos cognitivos estructurales no tienen la suficiente prueba concreta de que han sido estimulados, y si lo fueron, no lo hicieron con ese propósito sino por causas inespecíficas.

No obstante, se deben mencionar algunas limitaciones que se tuvo en el proceso investigativo; una de las restricciones del estudio radicó en el procedimiento de selección dela muestra, tratándose de un método no probabilístico, el grupo fue elegido con criterios de inclusión y exclusión. Este tipo de 
muestreo reduce en cierto grado la posibilidad de generalización de los resultados, sin embargo, es necesario matizar que los criterios mencionados, fueron definidos y seguidos de forma rigurosa. Otra limitante fue el tiempo ya que se trabajó de manera espaciada, dejando un entredicho sobre la capacidad misma de la cognición en el trabajo irrumpido. Otra limitante fue la evaluación del cociente intelectual, ya que otros estudios afirman que la RC es una ponderación proyectiva de la capacidad intelectual del individuo, por lo que este estudio se lo realizó a manera de impacto sin antecedentes intelectuales.

Sin duda alguna, el trabajo en la estimulación cerebral cognitiva y de la reserva cognitiva es un proceso positivo para todas las personas en edades tempranas y tardías, una correcta estimulación, estructuración y solidificación de la parte cognoscitiva de los individuos en crecimiento garantizan "salvo condiciones patológicas irreversibles" una calidad de vida en la adultez de mayor calidad ante el medio que frecuenta la persona, evitando una de la enfermedades comunes en la adultez que son las demencias y afines.

\section{CONCLUSIONES}

Las personas que en la adolescencia y adultez realizaron actividades sistemáticas como investigar, leer y aprendizaje de otros idiomas, además, de interacciones con juegos intelectuales como ajedrez, puzles, crucigramas y otros relacionados, generan una óptima reserva cognitiva que se transforman en factores protectores en la vejez, mejorando su calidad de vida en el envejecimiento. Considerando que en otras investigaciones suman criterios como actividades deportivas para fomentar la reserva cognitiva.

La educación básica y continua es un elemento indispensable y de extrema aplicabilidad para los individuos de cualquier grupo etario, en ella se cimientan todos los accesos al aprendizaje y conocimiento integral, estructurándole a la neurocognición los flujos suficientes para mantener los procesos activos de lo antiguo y nuevo, convirtiendo este accionar en determinante para los reservorios cognitivos que van a ser protectores en la vejez.

Factores relacionados a un bajo trabajo cognitivo en las diferentes etapas de su vida, condiciones ambientales desfavorables, y estos asociados a trabajos de oficio, manuales, monótonos y repetitivos, los cuales, no evidencian un abastecimiento a los niveles de reserva cognitiva, desencadenando una proclividad exponencial al pronto aparecimientos de declives cognitivos y patológicos, produciéndose procesos adversos en áreas familiares, sociales y económicas.

La reserva cognitiva es un mecanismo de influencia extra en la percepción de la calidad de vida de los seres humanos, convirtiendo al trabajo y estimulación cognitiva, una fuente preventiva de posibles patologías demenciales y coadyuvador al envejecimiento saludable de las personas; en medida que sus diversos componentes conducirán a la consecución de una capacidad neurofuncional adecuada y un equilibrio del estado cognitivo conectado a lo emocional y conductual.

REFERENCIAS

Ferrando, J., Nebot, M., Borrell, C. y Egea, L. (1996). Apoyo social y estado de salud percibido en población no institucionalizada de más de 60 años. Gaceta Sanitaria

Folstein, M. F., Folstein S. y Mchugh P.R. (1975). Mini-Mental State: A practical method for grading the cogntive state of patients for the clinicians. Journal of Psychiatric Research

Inouye, K. y Pedrazzani, E. S. (2007). Nivel de instrucción, socioeconómico y evaluación de algunas dimensiones de la calidad de vida de 
octogenarios. Revista Latino-Americana de Enfermagem

Rodríguez, M. y Sánchez, J. L. (2004). Reserva cognitiva y demencia. Anales de Psicología. p.20

Stern, Y. (2002). What is cognitive reserve? Theory and research application of the reserve concept. Journal of the International Neuropsychological Society. p. 448

Scarmeas, N. (2007). Patrones de estilo de vida y reserva cognitiva. En Y. Stern (Ed.), Reserva cognitiva: teoría y aplicaciones (págs. 187-206). Taylor y Francis.

Solé-Padullés, C., Bartr es-Faz, D., Junqu e, C., Vendrell, P., Rami, L., Clemente, IC, et al. (2009). Cerebro estructura $y$ función relacionado a cognitivo reserva variables en normal envejecimiento, leve cognitivo discapacidad y Alzheimer's enfermedad. Neurobiol. Envejecimiento 30 (7), 1114-1124. https://doi. org/10.1016/j.neurobiolaging.2007.10.008.

Valenzuela, J. (2008). Habilidades de pensamiento y aprendizaje profundo. Revista Iberoamericana De Educación, 46(7), 1-9. https://doi. org/10.35362/rie4671914

Zea-Herrera, M. C., López, M. E., Valencia, C. M., Soto, J. A., Aguirre, D. C., Restrepo, F. L., Oswald, W. D. y Rupprecht, R. (2008). Autovaloración de calidad de vida y envejecimiento en adultos con riesgo de Alzheimer. Investigación y Educación en Enfermería 